Sensors 2006, 6, 503-513

sensors

ISSN 1424-8220

(C) 2006 by MDPI

http://www.mdpi.org/sensors

Special Issue “Gas Sensors” Edited by Wenfeng Peng

Full Research Paper

\title{
CNT Sensors for Detecting Gases with Low Adsorption Energy by Ionization
}

\author{
Seongjeen Kim \\ Department of Electronic Engineering, Kyungnam University, Masan, Korea 631-701 \\ E-mail: sjk1216@kyungnam.ac.kr
}

Received: 29 March 2006 / Accepted: 4 May 2006 / Published: 5 May 2006

\begin{abstract}
In case of typical chemical gas sensors reacted by gas adsorption on surface of an active layer, it is difficult to detect some gases which have low chemical adsorption energy like inert gases. In this paper, we report a gas sensor using carbon nanotube(CNT) array as electron emitters for the purpose of detecting these gases. Specifically, sensors were fabricated with applications of glass patterning by a sand-blast process and of anodic bonding between glass and silicon to improve the compactness of the structure and the reliability in process. The proposed sensor, based on an electrical discharge theory known as Paschen's law, worked by figuring the changes of dark discharge current and initial breakdown voltage depending on the concentration and the identity of gases. In this work, air and Ar gases were examined and discussed.
\end{abstract}

Keywords: carbon nanotubes, gas sensor, Paschen's law, ionization.

\section{Introduction}

Chemical gas sensors usually utilize an electrical or optical response by adsorption of gas molecules on surface of an active layer. Among them metal-oxide gas sensors [1-3] are a representative example. The metal-oxide gas sensors consist of $\mathrm{SnO}_{2}, \mathrm{ZnO}$ or $\mathrm{TiO}_{2}$, as active layer, where the adsorption of gas molecules leads to a large change in its electrical resistivity. They have merits of low cost, good sensitivity, and convenient form of response (a simple change in resistance). But in order to achieve enhanced chemical reactivity between gas molecules and active layer, the sensors need to drive at high temperature of $200-500^{\circ} \mathrm{C}$ which causes problems of high power consumption and pre-heating time. Otherwise, some gas sensors which enable to operate even at room temperature have ever been developed with no metal-oxide like porous silicon [4-5]. However, all gas-adsorptive types of sensors 
have still potential difficulties in detecting gases with low adsorption energy or low electronegativity toward the active layer, among other limitations.

By the way, ionization sensors [6-8] which can detect gas molecules, regardless of the magnitude of the gas adsorption energy, have been deployed for a variety of applications such as environmental monitoring, sensing in chemical processing plants, and gas detection for counter-terrorism. They work by fingerprinting ionization characteristics of distinct gases. Generally the hot-cathode type of ionization sensors has been more prevailed due to easy thermionic emission of electrons from a cathode than the cold-cathode type by field emission, but the thermionic emission has some weak points; as pressure is very sensitive to temperature, the heat from filament must be blocked, and as chemical reaction by heated filament leads to additional contribution to the concentration, it must be restricted. Besides, all of the ionization sensors are limited by their huge and bulky architecture, high power consumption and risky high-voltage operation as they can be little fabricated by typical semiconductor process. If standard batch processing, as in the IC industry, is applicable to manufacturing sensors, a lot of identical sensors will be produced in one run, thus substantially improving their performance/cost ratio. Additionally, the miniaturization of sensors will not only contribute to their potentially low cost, but also allow them to be integrated with microelectronic circuits; thus, further enhancing their performance.

Here we propose a sensor differentiated from past ionization sensors [6-8] by its small size and by processes used in its manufacture. Recently, carbon nanotube(CNT)-based sensors which may be classified into chemical and physical types for convenience have been developed for gas detection. Early chemical types of CNT sensors were reported in some journals [9-12]. They usually figured out the data with the changes of conductivity and permittivity in CNTs by gas adsorption, and have shown merits such as good electrical response even at room temperature as well as a small size.

On the other hand, physical types of CNT gas sensors [13-14] were later introduced which utilized ionization of neutral gas by collisions with electrons under highly applied voltage. In general, physical gas sensors should be strong to high-voltage application. CNTs have relatively low electron escaping work and very sharp tip curvatures, and remain structurally stable under high electric fields [15], so the CNTs have been known to be the best field emitters over conventional field emitting metals such as Mo and W. Further, it has been known that vertically aligned CNT array covering a cathode is more compatible to produce a consistent nanometer-scale surface topology than conventional planar electrodes or micro-machined electron emitters. Therefore the CNT array is expected as a good cathode for electron emission, leading to dark discharge current more effectively.

In this paper, we introduced efficient process for fabricating CNT sensors with simplicity and compactness in the structure and reliability in process, inspected I-V properties in the dark discharge region with the sensors, and discussed the results with well-known Paschen's curves.

\section{Device Fabrication and Measurement}

\subsection{Device Fabrication}

The proposed sensor consists of three layers; emitter, spacer and anode. For the formation of multiwalled carbon nanotube (CNT) array on a highly doped 4-inch n-type (100) silicon substrate, Ti was first deposited on the Si substrate to improve adhesion and to prevent reaction of the metal catalyst 
with the substrate, and then Fe deposition was followed with an electron-beam evaporator where the Fe thin film as a catalyst metal was deposited to a thickness in the range of $10 \mathrm{~nm}$, and was prepatterned into the size of $3 \times 3 \mathrm{~mm}^{2}$ with conventional photolithography and a shadow mask to allow selective growth of CNTs. The growth of the vertically aligned CNT array was carried out with thermal CVD equipment. Pretreatment process was done for nucleation of the Fe film into the size of 10 to 30-nm-diameter in $\mathrm{NH}_{3}$ gas at 850 , and then the CNT array were grown with $\mathrm{C}_{2} \mathrm{H}_{2}$ source gas as a precursor in which $\mathrm{C}_{2} \mathrm{H}_{2}$ was supplied in a flow rate of $30 \mathrm{sccm}$ at the temperature of $850^{\circ} \mathrm{C}$ for 10 $\min$.

After making the CNT emitters, we prepared a spacer layer for insulation between the emitters and the anode. A flat pyrex glass plate with $500 \mu \mathrm{m}$ thickness as spacer layer was engraved for air-opening on the surface by a sand-blast process. The sand-blast process is currently used in manufacturing flat panel displays, silicon chips, and the patterning of various substrates such as glass, carbon and ceramic. It provides precise glass patterning in a resolution of $\mu \mathrm{m}$ range with a conventional photolithography. Using steps similar to those in integrated-circuit fabrication, vents were precisely shaped into V-groove. Finally, the silicon substrates for the CNT emitters and the anode, respectively, were combined into two steps with the patterned pyrex glass by an anodic bonding process at $400^{\circ} \mathrm{C}$. The anodic bonding between silicon substrate and pyrex glass is compatible to minimize thermal mismatch. Figure 1 shows an image of patterned pyrex glass combined with Si substrate grown by selective CNT array, where the area of the substrate was about $1.2 \times 1.2 \mathrm{~cm}^{2}$.

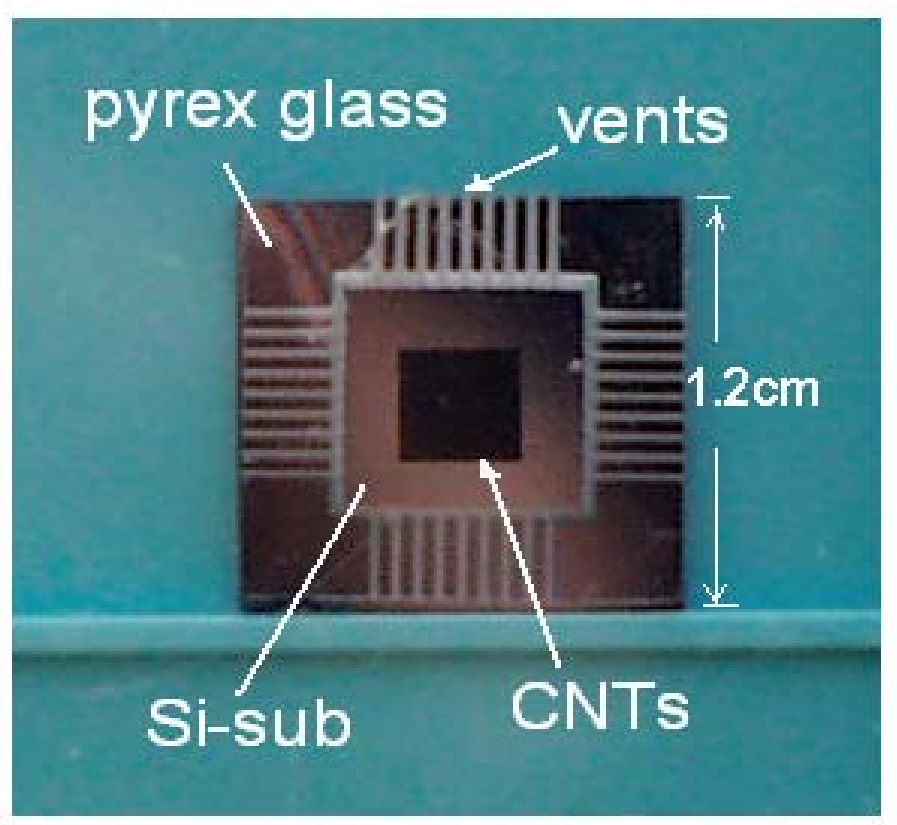

Figure 1. Image of pyrex glass and Si substrate after anodic bonding.

In this work, fabrication of the sensors was realized with a partial batch process on 4-inch wafers which is possible to the development of a full batch process eventually. The batch process provides merits such as reductions of the sensor size and the manufacturing cost, and the improved stability. 
Figure 2 shows a structure of the sensor and an anodic bonding procedure, and Figure 3 shows an image of a 4-inch patterned pyrex glass plate.

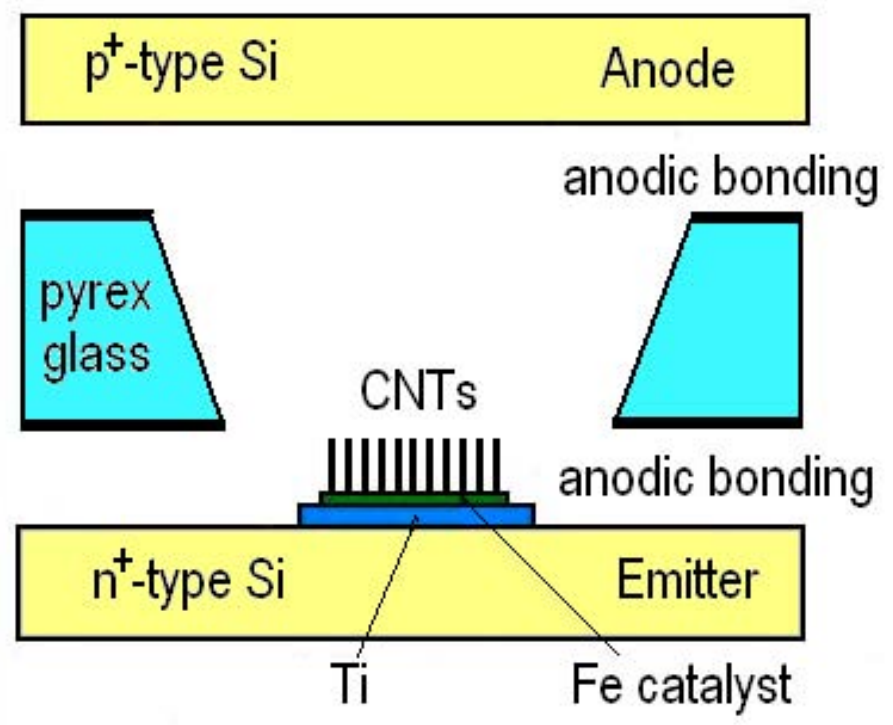

Figure 2. Schematic view of the sensor structure and an anodic bonding procedure.

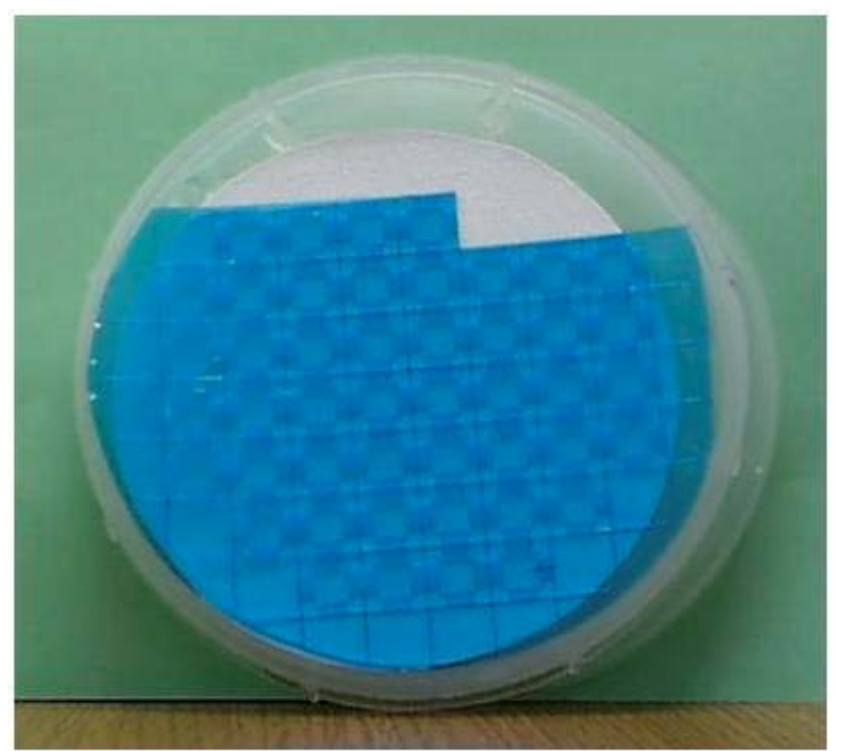

Figure 3. An image of a 4-inch patterned pyrex glass.

\subsection{Measurement}

The fabricated CNT sensor was placed for measurement in a chamber where gas could be pumped out up to the vacuum pressure of $1 \times 10^{-5}$ Torr. Silver paste contacts were made directly to both silicon 
plates of the sensors. The voltage was applied through electrical feed-through. All experiments were conducted at room temperature, and discharge current-voltage properties were measured with a high voltage source (Max. $1000 \mathrm{~V}$ corresponding to $2 \times 10^{6} \mathrm{~V} / \mathrm{m}$ ) and with Keithley 2425 SourceMeter as a current meter. In this work, we used air and Ar as target gases for testing the sensor. The gas concentration in the chamber was controlled from $10^{-7}$ to $10^{-4}$ [mol/liter] by pumping. The sensor was connected in series with a current-limiting resistance to restrict the electrical current. Figure 4 shows a diagram of experimental setup for testing the CNT sensor.

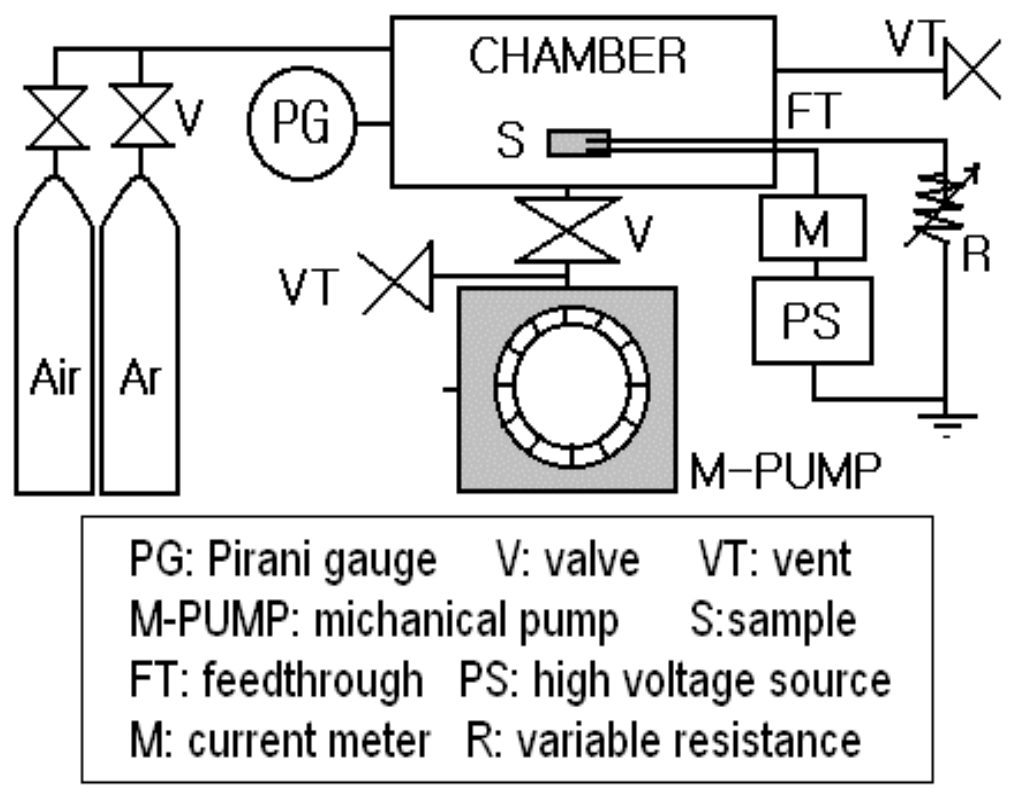

Figure 4. A diagram of experimental setup for testing the sensor.

\section{Results and Discussion}

Even though most gases in a chamber remain predominantly neutral in electricity, they can produce spontaneously a small number of electrons and ions by cosmic rays and natural radioactivity. Once these generated electrons and ions will move if a static voltage is applied; that is, electrons will migrate to an anode, ions to a cathode, and a small current will flow between two electrodes. But this current has no visible effects, and can be detected and measured only with difficulty because of small quantity in electrical charges.

By the electrical discharge theory in ideal gas, the typical discharge I-V curve is shaped as shown in Figure 5. When the applied voltage between an anode and a cathode is given as $V=V_{0}-I R$ where $V_{o}$ is the voltage supplied from the power supply, the I-V curve is divided to three parts and the operating point on the curve is determined by a load line according to values of the resistance, $R$. If operating point $\mathrm{A}$ is placed in a dark discharge region as shown in the figure, the current is no more than hundreds microampere, and is not self-sustained. If the applied voltage is increased or the resistance is 
reduced further, operating point A will move up the curve until the sparking voltage, $V_{s}$, is reached. And then the voltage applied to the sensor is sharply reduced, the operating point is transited to a glow discharge region, and the discharge is now self-sustained. If the resistance, $R$, is changed to a much smaller value in the glow discharge region, cathode heating may be enough to increase the electron supply and lower the discharge voltage, and the operating point will be transited to an arc region. Therefore, the transit of the operating point to other region on the discharge I-V curve is possible by controlling the applied voltage or the resistance.

In this work, the operating point of the sensor is limited only within the dark discharge region by connecting in series with a current-limiting resistance and by restricting the maximum applied voltage to $1 \mathrm{kV}$.

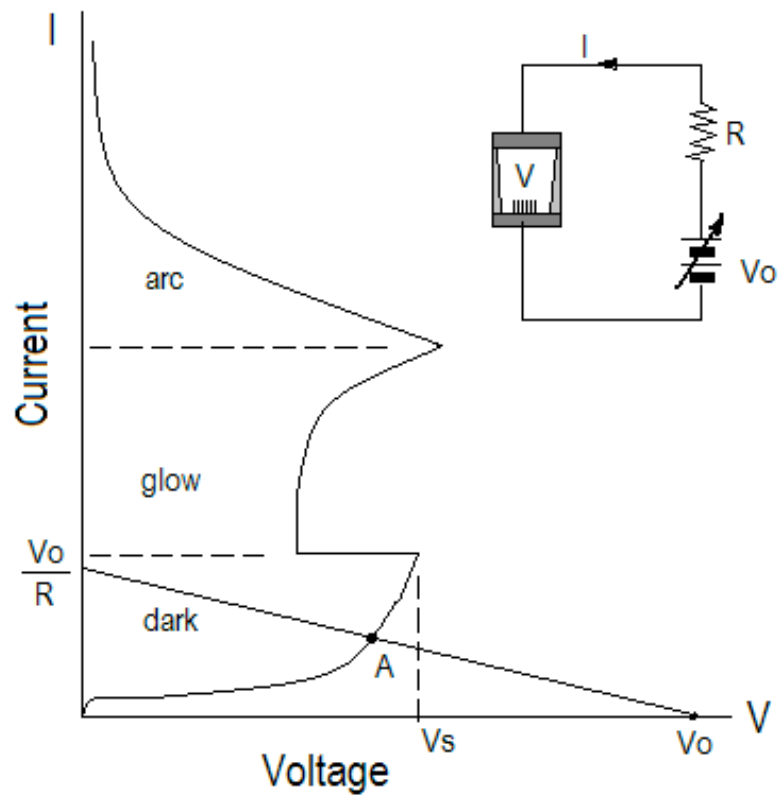

Figure 5. A typical discharge I-V curve in ideal gas.

We assume that the medium in the chamber is an ideal gas and that electrons are emitted from the micro tips of the vertically aligned CNT array under high applied voltage. Once electrons are emitted from the CNT array, they are accelerated towards the anode by electrical field, collide with gas molecules, and lead to the ionization of these neural molecules. Therefore, I-V characteristics in the dark discharge region depend both on the amount of electrons and positive ions generated through collisions and on the drift velocity of the electrons. Figure 6 shows the dependence of the dark current on air concentration in the chamber under the applied voltages of 300,500 and $1000 \mathrm{~V}$, where the concentration is defined as the unit of mol/liter and the distance between the electrodes is constant about $500 \mu \mathrm{m}$. As shown in the figure, the current increased with the applied voltage by the increase of the drift velocity, and it was found that the current increased abruptly over some critical concentrations on the voltages of 500 and $1000 \mathrm{~V}$, while any abruption in the current did not occur regardless of the concentration below the low applied voltage like $300 \mathrm{~V}$. That is, there existed a minimum voltage to 
cause a cascading of secondary electrons, since the electron energy gain is proportional to electrical field.

In summary, the dark current increased with the air concentration because more charges could be generated by collision, and the abruptions in current were shifted to the lower concentration as the static voltage increased higher. At $500 \mathrm{~V}$ the current abruption occurred at $5 \times 10^{-6} \mathrm{~mol} / \mathrm{liter}$, while at $1000 \mathrm{~V}$ it occurred at $1.5 \times 10^{-6} \mathrm{~mol} / \mathrm{liter}$.

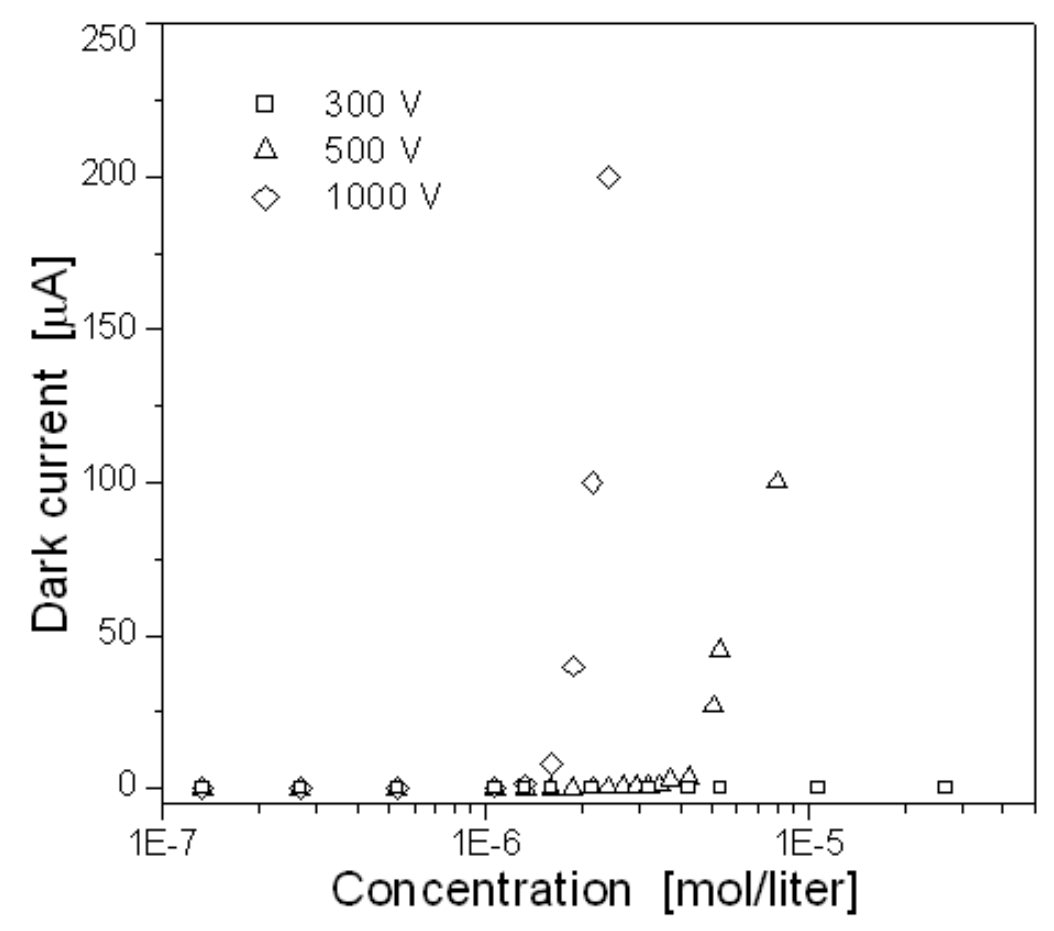

Figure 6. Dependence of the dark current on air concentration at different applied voltages.

In this work, we explain a detection process of the gas concentration and identity by ionization theoretically with Paschen's law. Paschen's law reflects a dark discharge mechanism, that is, a cascading of secondary electrons emitted by collisions in a gap, where the initial sparking breakdown voltage, $V_{s}$, is decided by a function of the product of the gas pressure, $p$, and the distance between two electrodes, $d$, and is expressed as

$$
V_{s}=f(\mathrm{pd})
$$

In this experiment, the breakdown voltage depends only on the pressure because the distance between the anode and the cathode is constant.

Paschen's law can be visualized graphically, where the curves show the lowest breakdown voltage to be roughly an order of magnitude larger than the ionization voltage. Starting from low pressure, the 
breakdown voltage at first decreases with increasing pressure because more molecules can be ionized, but at higher pressure the breakdown voltage increases with pressure because the mean free path decreases and electrons have difficulty reaching ionization energy.

By the way, the conversion from pressure to concentration can be derived by the state equation for ideal gas;

$$
\mathrm{p}=\mathrm{nRT} \text {, }
$$

where $\mathrm{p}$ is the pressure, $\mathrm{n}$ the concentration expressed as the number of moles of gas per unit volume, $\mathrm{R}$ the ideal gas constant, and $\mathrm{T}$ the absolute temperature.

An electron can ionize neutral atoms by collision with the energy required to free the least bound electron from an atom being the ionization energy. If the gas concentration changes, other parameters remaining constant, production of electron-ion pairs will change correspondingly. Figure 7 shows the dependence of the initial breakdown voltage on air concentration in the chamber. For simplification, the initial breakdown voltage was defined as the voltage necessary for $0.1 \mathrm{~mA}$ current. On the left side of the curve indicating the lower concentration, the initial breakdown voltage was fast increased with the reduction of the concentration because the dark discharge current depends on the amount of neutral molecules in the chamber which can provide electron-ion pairs generated by collisions. However, on the right side of the curve, the initial breakdown voltage was slowly increased with the increase of the concentration. In this region, the ionization rate is predominantly determined by the length of the mean free path rather than the amount of neutral molecules. The electron energy gain between two collisions is proportional to electrical field and is inversely proportional to concentration; thus if the gas concentration is higher, the mean free path will be shorter, and then the kinetic energy of the electron is not enough to ionize the neutral molecules. Therefore, as the ionization rate by collision is reduced with the concentration, the initial breakdown voltage increases with it. For air the minimum breakdown voltage was observed to be around $340 \mathrm{~V}$ under the condition that the distance between the electrodes was fixed to $500 \mu \mathrm{m}$.

The ionization rate of neutral molecules depends strongly on the concentration and the ionization energy of neutral molecules. Therefore the breakdown characteristics differ according to gases, and it is possible to detect the identity and the concentration of unknown gas species. Figure 8 shows the dependence of the breakdown voltage on Ar concentration. Over the whole range of the concentration the breakdown voltage in argon was lower than air because of relatively low electronegativity, even though the ionization energy of Ar gas $(15.7 \mathrm{eV})$ is somewhat larger than that of nitrogen $(15.5 \mathrm{eV})$ or oxygen $(12.6 \mathrm{eV})$ gases. In general, high electronegative gases such as $\mathrm{SF}_{6}$, Freon, oxygen, and nitrogen including air can reattach electrons after ionization easily and then turn to be neutral. Consequently, the discharge current in air is relatively reduced by the recombination, and the breakdown voltage in air becomes higher than that of Ar. The minimum breakdown voltage in $\mathrm{Ar}$ ambience was observed to be around $230 \mathrm{~V}$ less than that of air. 


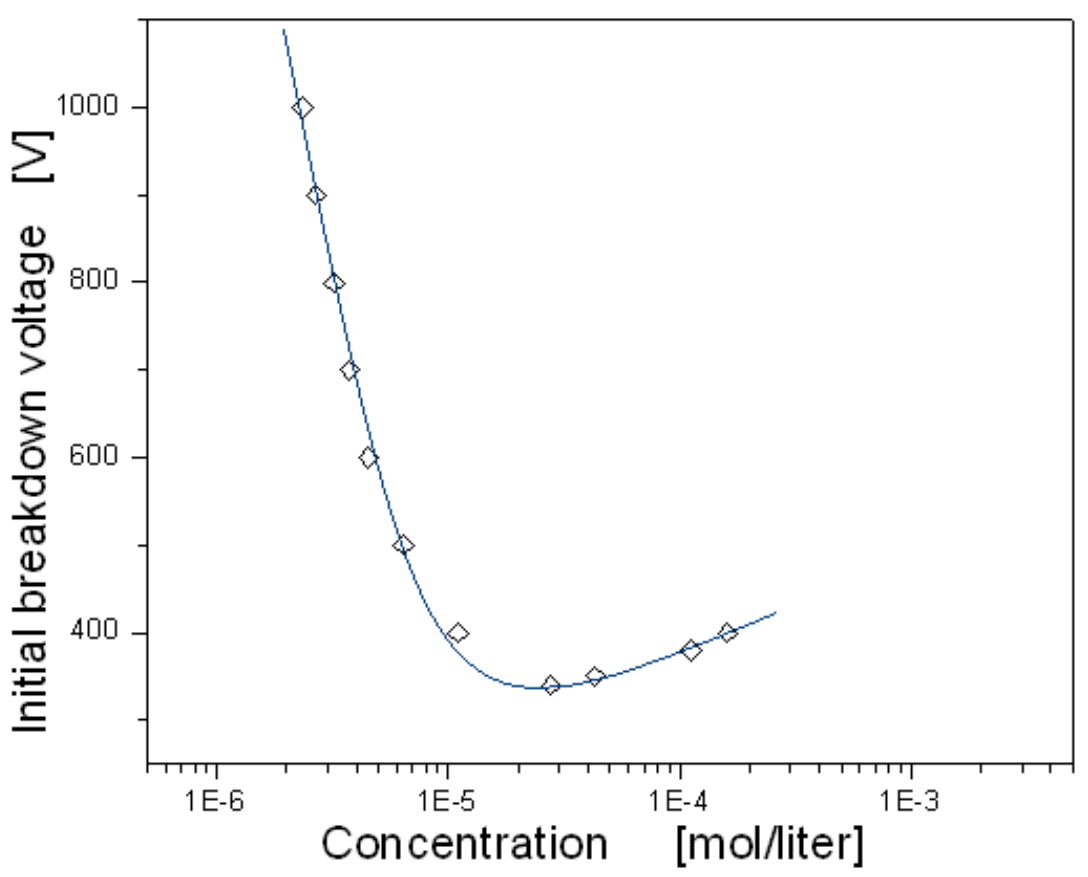

Figure 7. Dependence of initial breakdown voltage on air concentration.

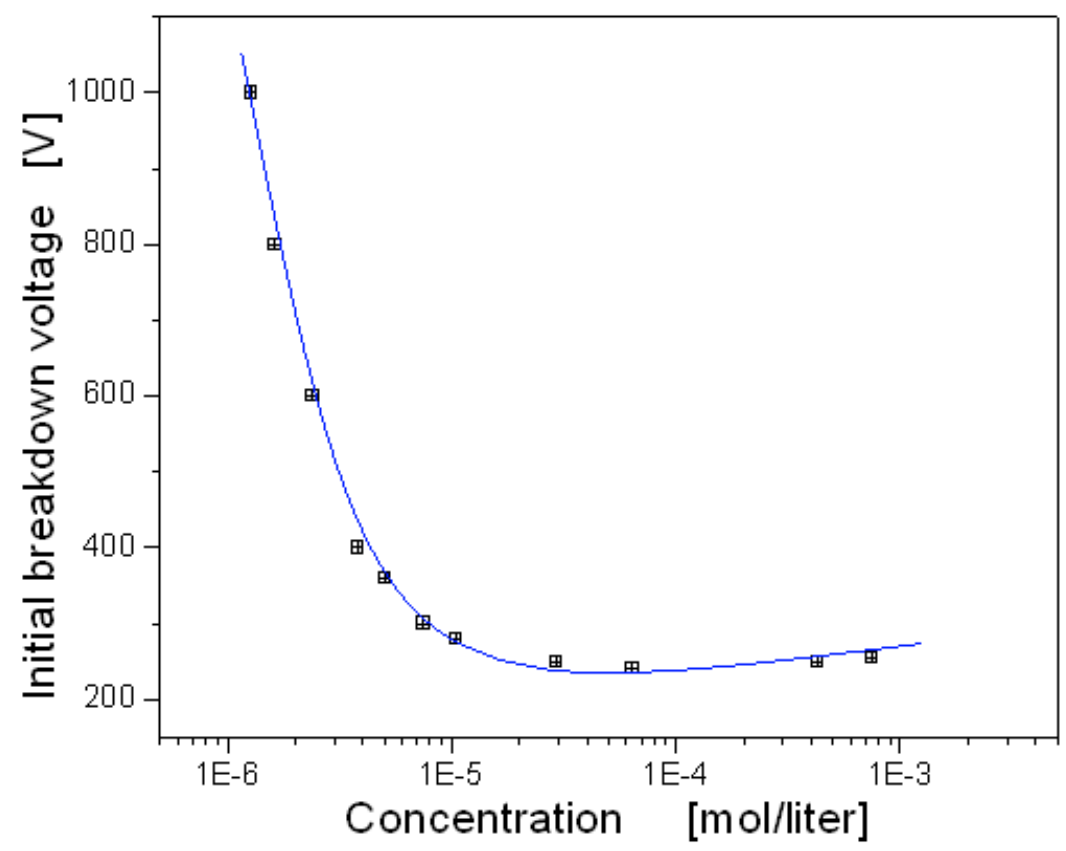

Figure 8. Dependence of initial breakdown voltage on Ar concentration.

\section{Conclusion}

In this work we reported an efficient fabrication and their performance of gas sensors using CNT array as electron emitters. By using the silicon-glass anodic bonding and glass patterning processes with the typical Si process, we improved the compactness of the structure and the reliability in process. The sensors have merits of small size, low cost, and low power consumption within tens mWs 
available to battery-power. In the experiment, breakdown characteristics in the dark discharge region were discussed as a function of gas concentration below the applied voltage of $1 \mathrm{kV}$ for air and Ar gases. As the result, even though it should be realized that there are many other factors which have an effect on the breakdown of a gap, the sensors led to similar result as predicted by Paschen's law. We expect that they are suitable to sensing vacuum pressure, environmental monitoring, sensing in chemical processing plants, and detecting inert gases.

\section{Acknowledgements}

This study was supported by the academic research fund of Kyungnam University in the year of 2005.

\section{References and Notes}

1. Varghese, O.; Grimes, C. Metal oxide nanoarchitectures for environmental sensing. J. Nanosci. Nanotechnol. 2003, 3, 277-293.

2. Shimizu, Y.; Egashira, M. Basic aspects and challenges of semiconductor gas sensors. Mater. Res. Soc. Bull. 1999, 24, 18-24.

3. Lee, M.; Meyer, J. A new process for fabricating CO sensing layers based on BaTiO and additives. Sensors and Actuators B 2000, 68, 293-299.

4. Watanabe, K.; Okada, T.; Choe, I.; Sato, Y. Organic vapor sensitivity in a porous silicon device. Sensors and Actuators B 1996, 33, 194-197.

5. Kim, S. J.; Lee, S. H.; Lee, C. J. Organic vapor sensing by current response of porous silicon layer. J. of Phys. D 2001, 34, 3505-3508.

6. Sedlacek, M. Electron Physics of Vacuum and Gaseous Devices; John Wiley \& Sons: New York, 1996; pp 386-400.

7. Abbott, P. J.; Looney, J. P.; Mohan, P. The effect of ambient temperature on the sensitivity of hotcathode ionization gauges. Vacuum 2005, 77, 217-222.

8. Akimichi, H.; Tanaka, T.; Takeuchi, K.; Tuzi, Y.; Arakawa, I. Development of a new ionization gauge with Bessel Box type energy analyzer. Vacuum 1995, 46, 749-752.

9. Kong, J.; Franklin, N.; Zhou, C.; Chapline, M.; Peng, S.; Cho, K.; Dai, H. Nanotube molecular wires as chemical sensors. Science 2000, 287, 622-625.

10. Varghese, O. K.; Kichambre, P. D.; Gong, D.; Ong, K. G.; Dickey, E. C.; Grimes, C. A. Gas sensing characteristics of multi-wall carbon nanotubes. Sensors and Actuators B 2001, 81, 32-41

11. Ong, K.; Zeng, K.; Grimes, C. A wireless, passive carbon nanotube-based gas sensor. IEEE Sensor J. 2002, 2, 82-88.

12. Jang, Y. T.; Moon, S. I.; Ahn, J. H.; Lee, Y. H.; Ju, B. K. A simple approach in fabricating chemical sensor using laterally grown multi-walled carbon nanotubes. Sensors and Actuators $B$ 2004, 99, 118-122.

13. Modi, A.; Koratkar, N.; Lass, E.; Wie, B.; Ajayan P. Miniaturized gas ionization sensors using carbon nanotubes. Nature 2003, 424, 171-174. 
14. Gavrilov, S. A.; Il’ichev, E. A.; Poltoratski, E. A.; Rychkov, G. S.; Dvorkin, V. V.; Dzbanovsky, N. N.; Suetin, N. V. Carbon nanotube field emitters for planar emission vacuum micro- and nanoelectronics. Tech. Phys. Lett. 2004, 30, 609-611.

15. Heer, W.; Chatelain, A.; Ugarte, D. A carbon nanotube field-emission electron source. Science 1995, 270, 1179-1180.

(C) 2006 by MDPI (http://www.mdpi.org). Reproduction is permitted for noncommercial purposes. 